\title{
Peculiarities of detecting Teflon defect surrogates in CFRP by transient IR thermography
}

\author{
by V. Vavilov*, G. Xingwang ${ }^{* *}$, S. Wei ${ }^{* *}$ and L. Yingtao** \\ * Tomsk Polytechnic University, Russia 634028, Tomsk, 28, Savinykh St., 7 \\ Tel./Fax +7 (3822) 4187 12, E-mail: vavilov@introscopy.tpu.ru \\ ** Beihang University, School of Mechanical Engineering and Automation, \\ Beijing 10083, P.R. China, Email: xingwangguo@hotmail.com
}

\begin{abstract}
A new thermal NDT model is proposed to explain a 'non-classical' behavior of temperature signals over Teflon inserts in CFRP. Simulation results are compared to experimental data suggesting that composite properties are modified near inserts.
\end{abstract}

\section{Introduction}

IR Thermographic Nondestructive Testing is a promising technique in the inspection of Carbon Fiber Reinforced Plastic (CFRP) [1-3]). In this study, we come back to the detection of Teflon inserts, which are often used as CFRP defect surrogates. Our experiments revealed the 'non-classical' behavior of surface temperature signals $\Delta T$ over Teflon inserts ( $\Delta T=T_{d}-T_{n d}$, where $T_{d}, T_{n d}$ are the temperatures in a defect and non-defect area respectively). In particular, negative $\Delta T$ values appeared instead of expected positive.

\section{Reference sample and experimental results}

The two CFRP reference samples A and B contained four and five Teflon inserts respectively at the depths of 1.3 and $0.5 \mathrm{~mm}$ (see figure 1). The samples were heated by two $3.5 \mathrm{~kJ}$ flash tubes and image sequences were captured by a Thermovision 900 system ( $15 \mathrm{~Hz}$ frame frequency). The sample excess temperature reached its maximum value of $100^{\circ} \mathrm{C}$ at the end of heating and decayed up to $21^{\circ} \mathrm{C}$ in the next image recorded at $0.067 \mathrm{~s}$ later. This ratio has allowed us to estimate the sample diffusivity as $a=3.25 \cdot 10^{-7} \mathrm{~m}^{2} / \mathrm{s}$ by using the ThermoCalc-2DM program. The absorbed power density was found to reach $Q_{m} \sim 1.510^{6} \mathrm{~W} / \mathrm{m}^{2}$.

In the sample $\mathbf{B}$, where defect depths were $0.5 \mathrm{~mm}$, up to three of five defects were reliably detected by simply looking through the sequence. In the sample $\mathbf{A}$, where defect depths were $1.3 \mathrm{~mm}$, the temperature behaved in a 'non-classical' way. Both detection situations can be described by the maximum temperature signal $\Delta T_{m}$ and the best observation time $\tau_{m}$. The corresponding estimates are given in table 1 .

\section{Modeling experimental temperature evolutions}

If there would be an ideal thermal contact between the Teflon and the host material, the $\Delta T$ behavior in the sample $\mathbf{A}$ would be 'classical'. This means that 
defects with the conductivity lower than that of CFRP will appear as warm spots of whose amplitudes will decay with growing defect depths, while the best observation times will become longer. The temperature inversion phenomenon described by some authors [3] has seemed to be improbable in our experiment because it assumes that a negative $\Delta T_{m}$ signal must be preceded by a classical 'warm' signal that is not our case. Therefore, our task was to choose a model that would fit both the negative temperature signal in the sample $\mathbf{A}$ and the positive signal in the sample $\mathbf{B}$.

It is known that inserting Teflon could introduce air gaps between inserts and a host material [3]. Therefore, Teflon inserts in CFRP are fairly well detected, although the theory predicts rather low signals in the case of an ideal thermal contact between an insert and a host material. We have assumed that compressing CFRP during manufacturing may increase the composite density $\rho$ across defect areas

thus increasing its conductivity $\lambda$. To simulate such model, we have also used the Thermocalc-2DM program. After multiple trials, we have come to the models of the sample $\mathbf{A}$ and $\mathbf{B}$ shown in figure $2 \mathrm{a}$. The calculated temperature profiles are shown in figure $2 \mathrm{~b}, \mathrm{c}$ and the corresponding $\Delta T_{m}$ and $\tau_{m}$ values are given in table 1.

It is important that only the CFRP thermal conductivity should be increased by $25 \%$ (from $\lambda=0.64 \mathrm{~W} /(\mathrm{m} \cdot \mathrm{K})$ to $\lambda=0.8 \mathrm{~W} /(\mathrm{m} \cdot \mathrm{K})$ ) with the thermal diffusivity $a$ being unchanged. It seems that the increases in both $\lambda$ and $\rho$ may compensate each other due to the definition: $a=\lambda /(C \rho)$, where $C$ is the heat capacity that is constant under various material modifications. Another assumption has been that conductivity modifications touch only the layers adjacent to Teflon inserts.

Decent verification of the proposed model has not been available because we have had no Contractor's permission to disassemble the reference samples. Therefore, we consider the proposed model as preliminary.

\section{Image processing}

In this study, we have processed the experimental results by using the approach essentially based on applying some statistical procedures [3]. Each image is characterized by the signal-to-noise ratio (ThermoStat program):

$$
S N R=\left(\bar{T}_{d}-\bar{T}_{n d}\right) / \sigma_{n d},
$$

where $\bar{T}_{d}, \bar{T}_{n d}$ are the mean temperatures in the defect and non-defect areas respectively, $\sigma_{n d}$ is the temperature standard deviation in a non-defect area. The probabilities of correct detection $P_{c . d \text {. }}$ and false alarm $P_{f . a}$. can be determined by the corresponding histograms. The chosen defect areas have covered a priori known defects, while the rest of the sample has been considered sound.

For the sample $\mathbf{A}$, the processing results have been rather discouraging. Only the use of the Fourier transformation has allowed enhancing the SNR from 0.5 to 1.5 (compare the source image and the phasegram in figure 3). Note that in this case the chosen defect areas have covered only two visible defects.

The bad results obtained on the sample A can be explained by too short heating that has lead to the sample temperature close to the ambient one quickly 
after heating. It was reported elsewhere [3] that the defects in a similar CFRP sample were detected at the depths up to $4 \mathrm{~mm}$ due to the longer heat pulse $(4.4 \mathrm{~s})$.

In their turn, the results obtained on the sample $\mathbf{B}$, can serve as a good illustration of the efficiency of some processing algorithms (see table 2 and figure 4). We have used the following techniques realized in the ThermoFit Pro program: 1) Fourier transformation in time, 2) thermal tomography (maxigrams and timegrams), 3) polynomial fitting, 4) normalization, and 5) correlation. All techniques are well-known, except correlation.

In the case of sample B, chosen defect areas have covered all five defects. To surprise, the highest $S N R=39.6$ value has been provided by the image, where the correlation between a chosen reference pixel and other pixels has been calculated. The peculiarities of this technique are not well-explored, so we make no comments on the obtained $S N R$ value (except the fact that all correlation coefficients were very close to unity and there might be the calculation accuracy problem while determining the signal standard deviation).

In many images, three defects of the diameter from 20 to $5 \mathrm{~mm}$ are reliably detected. Some images show clear indications in the area of the smallest defect (see the phasegram in figure 4c). The use of the defect characterization algorithm incorporated in ThermoFit Pro program proved to be also efficient. The 'depthgram' shown in figure $4 d$ demonstrates zero pixel values in non-defect areas and from 0.44 to $0.55 \mathrm{~mm}$ values over all defects that is close to the true value of $0.5 \mathrm{~mm}$. The ThermoFit Pro program also allows determining defect thermal resistance that has been about $R_{d}=d / \lambda_{d}=(2.3-3) \cdot 10^{-3} \mathrm{~W}^{-1} \mathrm{~m}^{2} \cdot \mathrm{K}$ in our case, while the model in figure $2 \mathrm{a}$ implies the total thermal resistance of air and Teflon to be $1.83 \cdot 10^{-3} \mathrm{~W}^{-}$ ${ }^{1} \mathrm{~m}^{2} \cdot \mathrm{K}$. It is worth noting that the accuracy in evaluating $R_{d}$ or $d$ is typically low.

Finally, some images have been used to produce binary maps of defects (figure 5). It is seen that, if to apply the optimal detection technique, i.e. to use the source image at the optimal observation time ( $0.8 \mathrm{~s}$ for the sample $\mathrm{B}$ ), the detection limit is characterized by defect diameters about $10 \mathrm{~mm}$ (only two defects are seen in the map of figure 5a). This case is characterized by the following probabilities: $P_{c . d .}=86.8 \%$ and $P_{f . a .}=5 \%$. The map shown in figure $5 \mathrm{~b}$ demonstrates that, by applying the correlation technique, it is possible to detect a smallest defect by the diameter of $3 \mathrm{~mm}\left(P_{c . d .}=99.8 \%\right.$ and $\left.P_{f . a .}=5 \%\right)$.

\section{Conclusions}

- The experimental results obtained on the CFRP reference samples containing Teflon inserts have revealed the 'non-classical' behavior of surface temperature gradients leading to negative $\Delta T_{m}$ values for the defects at the depth of $\sim 1.3 \mathrm{~mm}$, while, in the case of shallower defects, the signals proved to be close to those predicted by a simple (classical) mathematical model.

- The discrepancy between experimental and expected results has been overcome by proposing the sample model where Teflon inserts are surrounded by air gaps and the density and conductivity of a host composite is modified through the layers adjacent to the inserts. Within such model, the calculated $\Delta T_{m}$ and $\tau_{m}$ values proved to be close to those observed in the experiment. 
- Several processing algorithms have been statistically evaluated to demonstrate that both the Fourier transformation and the novel correlation technique provide the highest values of signal-to-noise ratio.

- It can be stated that, by using flash heating and looking at the source image at the best observation time, it is possible to detect defects with the diameter greater than $10 \mathrm{~mm}$ at the depth of $0.5 \mathrm{~mm}$. The use of advanced data treatment algorithms reduces this test limit up to defects greater than $3 \mathrm{~mm}$ in the diameter. However, flash heating seems to be inefficient in detecting deeper defects; it is believed that longer heat pulses will be more appropriate in this case.

\section{REFERENCES}

[1] Balageas, D.L. and Deom, A.A. Characterization and NDT of carbon epoxy composites by a pulsed photothermal method, Mater. Evaluation, P. 461-465 (April 1987).

[2] Bendada, A., Maillet, D. and Degiovanni, A. Nondestructive transient thermal evaluation of laminated composites: discrimination between delaminations, thickness variations and multidelaminations, Proc. "Quant InfraRed Thermography QIRT'92”, P. 218-223, Châtenay-Malabry, France (1992).

[3] Vavilov, V., Almond, D.L., Busse, G. et al. Infrared thermographic detection and characterization of impact damage in carbon fiber composites: Round robin test results, Proc. "Quant. InfraRed Thermography QIRT'98", P. 33-40, Lodz, Poland (1998).

Table 1. Experimental and simulated model parameters in the inspection of CFRP*

\begin{tabular}{|c|c|c|c|c|}
\hline \multicolumn{2}{|c|}{$\begin{array}{c}\text { Detection } \\
\text { parameters }\end{array}$} & $\begin{array}{c}\text { Experimental } \\
\text { values }\end{array}$ & $\begin{array}{c}\text { Classical model } \\
\text { (Teflon insert, ideal contact) }\end{array}$ & $\begin{array}{c}\text { Model by } \\
\text { figure 2a }\end{array}$ \\
\hline Sample A & $\Delta T_{m}$ & $\sim-0.2^{\circ} \mathrm{C}$ & $+0.21^{\circ} \mathrm{C}$ & $-0.2^{\circ} \mathrm{C}$ \\
\cline { 2 - 5 } & $\tau_{m}$ & $\sim 1-2 \mathrm{~s}$ & $2.23 \mathrm{~s}$ & $1.1 \mathrm{C}$ \\
\hline Sample B & $\Delta T_{m}$ & $+1.2^{\circ} \mathrm{C}$ & $+0.81^{\circ} \mathrm{C}$ & $+1.3^{\circ} \mathrm{C}$ \\
& $\tau_{m}$ & $0.8 \mathrm{~s}$ & $0.63 \mathrm{~s}$ & $0.78 \mathrm{~s}$ \\
\hline
\end{tabular}

* Thermal properties of CFRP: $\lambda=0.64 \mathrm{~W} /(\mathrm{m} \cdot \mathrm{K}), a=0.52 \cdot 10^{-6} \mathrm{~m}^{2} / \mathrm{s}$;

Teflon: $\lambda=0.25 \mathrm{~W} /(\mathrm{m} \cdot \mathrm{K}), a=1.1 \cdot 10^{-6} \mathrm{~m}^{2} / \mathrm{s}$; air: $\lambda=0.07 \mathrm{~W} /(\mathrm{m} \cdot \mathrm{K}), a=5.8 \cdot 10^{-5} \mathrm{~m}^{2} / \mathrm{s}$

Table 2. Statistical treatment results (sample B)

\begin{tabular}{|l|c|}
\hline \multicolumn{1}{|c|}{ Image } & Signal-to-noise ratio \\
\hline Correlation image (normalized sequence) & 39.6 \\
\hline Image of the $\mathrm{A}_{2}$ polynomial coefficient & 7.7 \\
\hline Phasegram at $\mathrm{f}_{2}=0.15 \mathrm{~Hz}$ & 6.0 \\
\hline Phasegram at $\mathrm{f}_{3}=0.3 \mathrm{~Hz}$ & 5.8 \\
\hline Best source image after fitting and normalization $(\tau=0.8 \mathrm{~s})$ & 5.4 \\
\hline Maxigram after fitting and normalization & 5.3 \\
\hline Phasegram at $\mathrm{f}_{4}=0.45 \mathrm{~Hz}$ & 4.3 \\
\hline Best source image after normalization $(\tau=0.8 \mathrm{~s})$ & 2.6 \\
\hline Best source image $(\tau=0.8 \mathrm{~s})$ & 2.0 \\
\hline Image at the end of heating & 0.1 \\
\hline
\end{tabular}




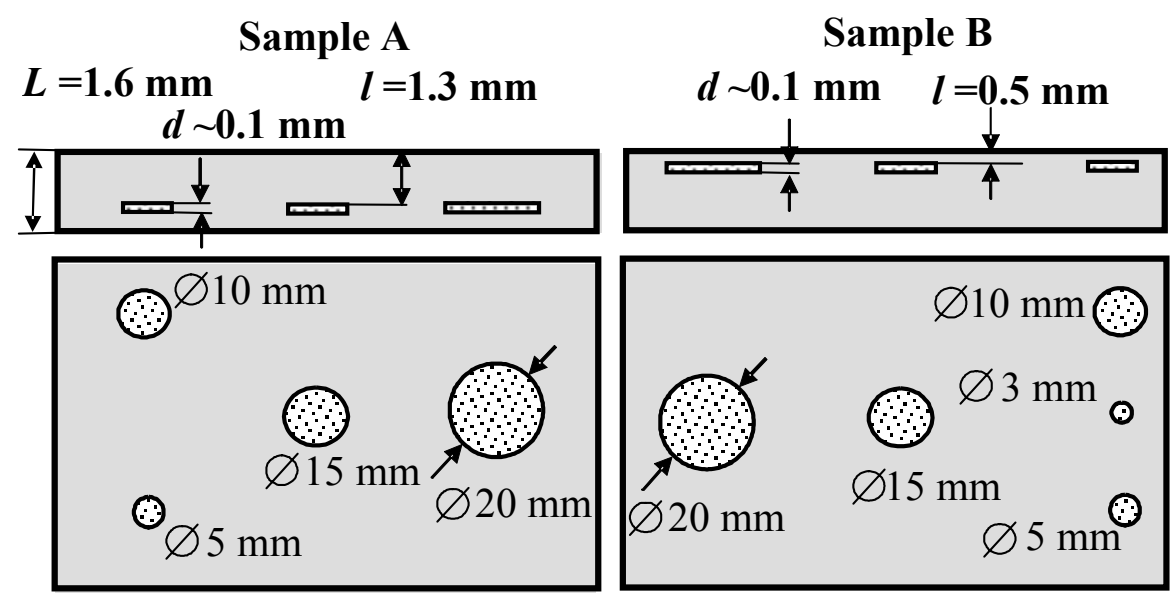

Fig. 1. CFRP reference sample scheme
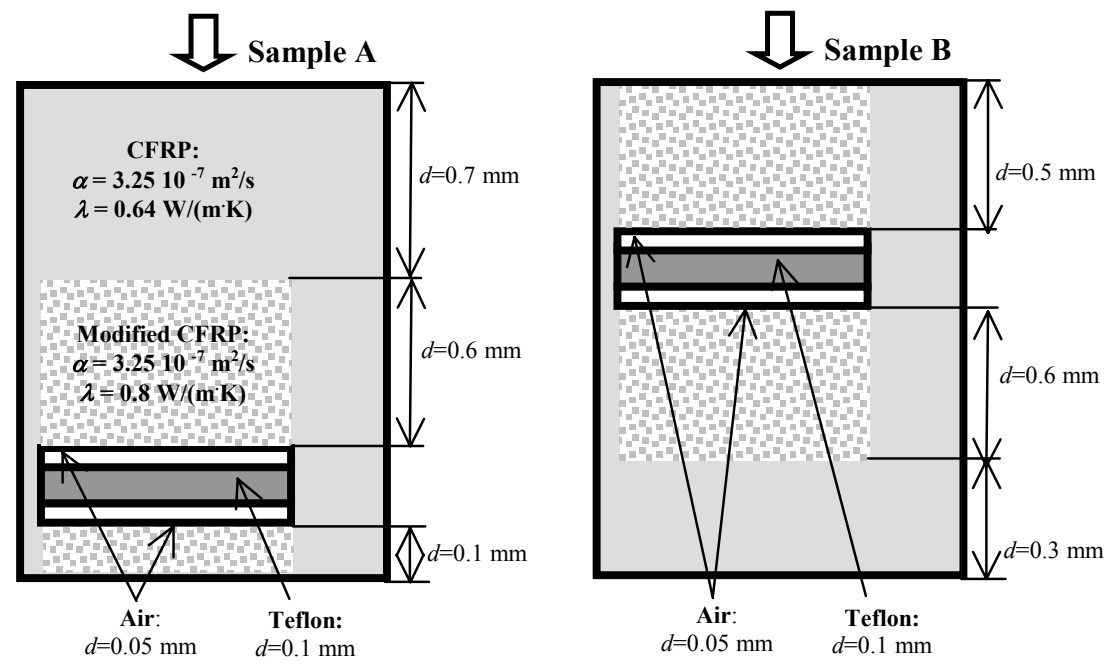

a)

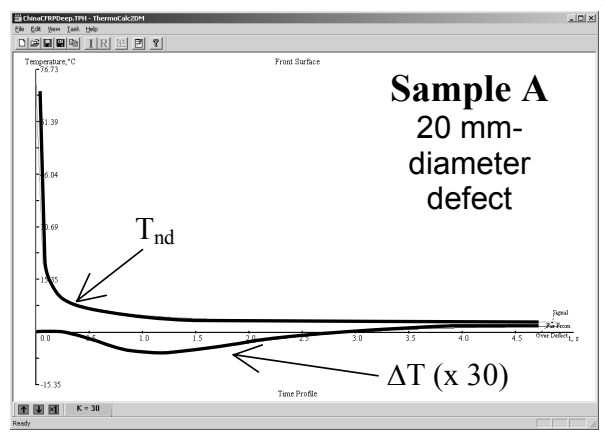

b)

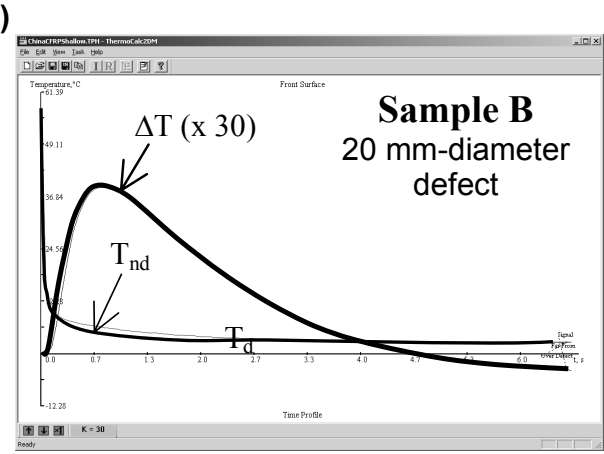

c)

Fig. 2. Modeling thermal NDT of CFRP 


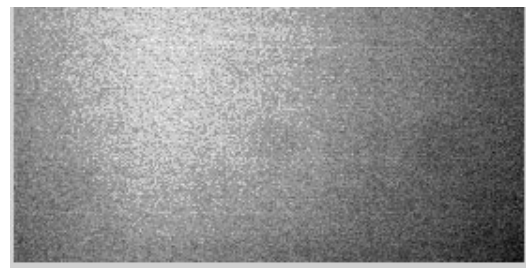

a)

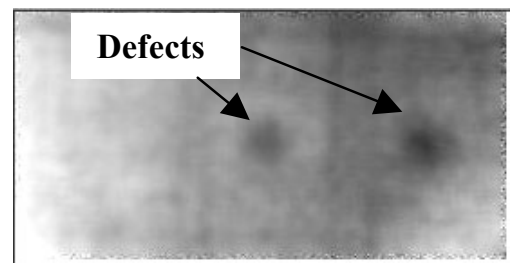

b)

Fig. 3. Image processing results (sample A):

a - 'best' source image ( $S N R=0.5)$,

$\mathrm{b}$ - phasegram at $0.15 \mathrm{~Hz}$ after smoothing $7 \times 7(S N R=1.5)$

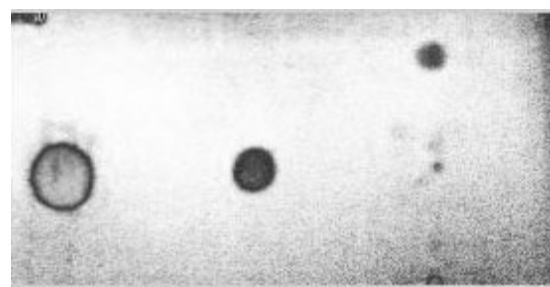

a)

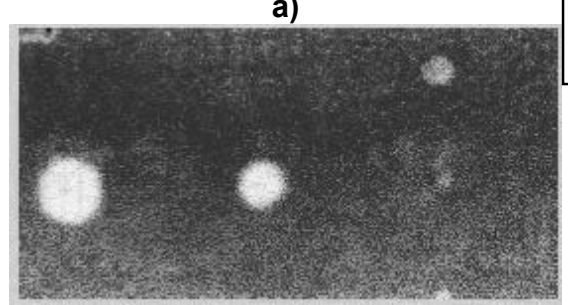

c)

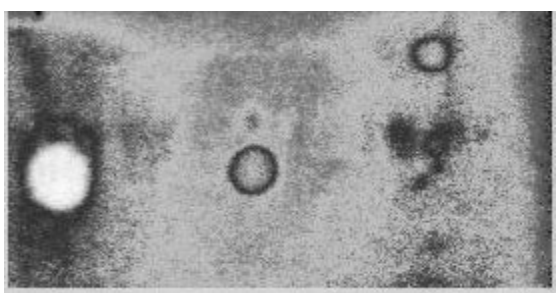

b)

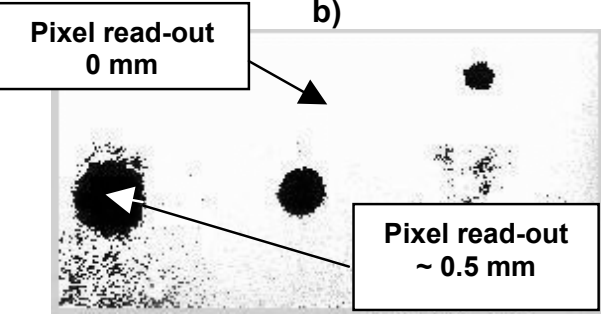

d)

Fig. 4. Image processing results (sample $\mathbf{B}$ ):

a - correlation image (fitted normalized sequence), $S N R=39.6$,

$\mathrm{b}$ - phasegram at $\mathrm{f}_{3}=0.3 \mathrm{~Hz}, S N R=5.8$,

$\mathrm{c}$ - best source image after normalization ( $\tau=0.8 \mathrm{~s}$ ), $S N R=2.6$,

$\mathrm{d}$ - depthgram (threshold established)

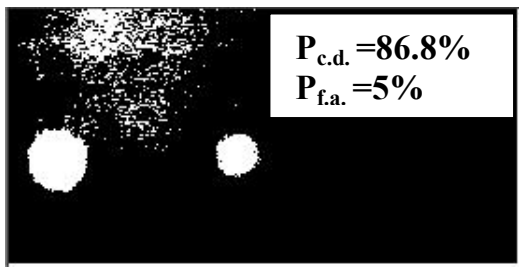

a)

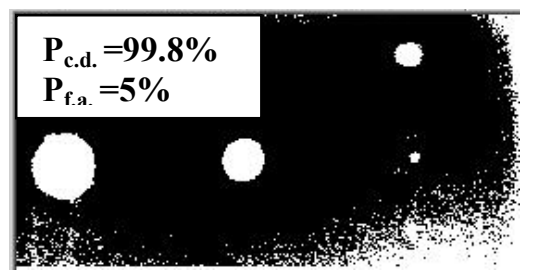

b)

Fig. 5. Statistical maps of defects (sample B):

a - by the raw image captured at the optimal observation time $\left(\tau_{m}=0.8 \mathrm{~s}\right)$,

$\mathrm{b}$ - by the correlation image (raw normalized sequence) 